\title{
Transcritical mixing of sprays for multi-component fuel mixtures
}

\author{
Julien Manin*1,2, Cyril Crua ${ }^{3}$, Lyle M. Pickett ${ }^{1}$ \\ ${ }^{1}$ Combustion Research Facility, Sandia National Laboratories, Livermore CA, USA \\ ${ }^{2}$ Artium Technologies, 470 Lakeside Dr., Sunnyvale CA, USA \\ ${ }^{3}$ Advanced Engineering Centre, University of Brighton, Brighton BN2 4GJ, United Kingdom \\ ${ }^{*}$ Corresponding author: imanin@artium.com
}

\begin{abstract}
The mixing of fuels with oxidizer has been an increasingly interesting area of research with new engine technologies and the need to reduce emissions, while leveraging efficiency. High-efficiency combustion systems such as diesel engines rely on elevated chamber pressures to maximize power density, producing higher output. In such systems, the fuel is injected under liquid state in a chamber filled with pressurized air at high temperatures. Theoretical calculations on the thermodynamics of fuel mixing processes under these conditions suggest that the injected liquid can undergo a transcritical change of state. Our previous experimental efforts in that regard showed through highspeed imaging that spray droplets transition to fluid parcels mixing without notable surface tension forces, supporting a transcritical process. Only mono-component fuels were used in these studies to provide full control over boundary conditions, which prevented extrapolation of the findings to real systems in which multi-component fuels are injected. Multi-component fuels add another layer of complexity, especially when detailed experiments serve model development, requiring the fuels to be well characterized. In this work, we performed high-speed microscopy in the near-field of high-pressure sprays injected into elevated temperature and pressure environments. A reference diesel fuel and several multi-component surrogates were studied and compared to single component fuels. The results support that a transition occurs under certain thermodynamic conditions for all fuels. As anticipated, the transition from classical evaporation to diffusive mixing is affected by ambient conditions, fuel properties, droplet size and velocity, as well as time scales. Analogous to previous observations made with the normal alkane sprays, the behavior of the multi-component fuels correlate well with their bulk critical properties.
\end{abstract}

\section{Keywords}

Diesel sprays, Transcritical mixing, Multi-component fuels.

\section{Introduction}

Despite the growth of alternative technologies for power generation in transportations systems, these technologies only represent a fraction of the worldwide vehicle fleet. Forecasts predict that thermal engines are still going to be highly dominant in decades to come [1]. The same predictions expect a $75 \%$ increase in fuel demand between air, marine and rail transportation, while heavy-duty alone would account for $40 \%$ of the total transportation energy demand. Even with the substantial improvements in gasoline direct-injection and the development of promising new combustion strategies (e.g., homogeneous-charge compression-ignition, spray-guided spark ignition or gasoline compression-ignition), heavy-duty, marine and rail transportation are expected to rely on diesel combustion for years to come. The mixing process between the injected fuel and the oxidizer in diesel engines has been long identified as a key parameter to efficient and clean combustion. Experiments have been devoted to understanding mixing under such conditions, but gaps remain regarding the vaporization process at these extreme thermodynamic conditions. Further, the high velocities and small flow scales produced by the elevated injection pressures limit our ability to fully study and understand the complex processes at play in the confined environment of an engine's combustion chamber.

A substantial part of the research over the last decade has been dedicated to build computational fluid dynamic models to understand these processes. One major limitation is encountered when the fundamental knowledge about the physics of the process to be numerically predicted is inaccurate or incomplete. Such an issue concerns the multi-phase mixing process under elevated pressures and temperatures. Recent theoretical studies aiming at resolving the interface between the liquid and the ambient gas under diesel-like conditions, as well as the state of the mixture, suggest that the high pressures and temperatures the injected fluid is subjected to may broaden this interface [2,3]. As a result, the surface tension between the two fluids may decrease dramatically to the point that the interface enters the continuum regime. Under such molecular state, the integrity of the interface may easily be challenged by external forces such as aerodynamic or turbulence, potentially leading to a single-phase type of mixing. The conditions found in diesel engines are favorable to such process, analogous to the transcritical mixing observed in cryogenic liquid injection applied to liquid rockets $[4,5]$. 
Beyond studies carried out by the rocket community at very different conditions from those of diesel engines, detailed experiments convincingly showing the transcritical mixing process of spray droplets were inexistent until the recent experiments performed by the authors [6]. Other works [7, 8] and past efforts by our group [9] suggested the different mixing behavior based on macroscopic observations or optically-degraded microscopic features. But they failed at capturing the mixing process at the droplet scale, thus leaving room for controversy. The authors' most recent work [6] succeeded in visualizing the transition from classical evaporation to diffusive mixing at the droplet level. The analysis of the high-speed visualizations revealed that the mixing processes could be classified into three regimes. It was confirmed that the classical perspective on evaporation and mixing occurred well beyond the critical point of the injected fluid, with spherical droplets presenting a surface-based vaporization process. Two new regimes were identified, where vaporization and mixing were accelerated due to fluid structure deformation and stretching or a behavior resembling a single-phase mixing process. Both these regimes seem to be affected by a severe drop in intermolecular forces. These two regimes were classified into transitional mixing and diffusive mixing, respectively. Transitional mixing was defined when the fluid structures were seen to deform and oscillate, contributing to an accelerated vaporization. Diffusive mixing corresponds to a regime where the fluid features eventually stretch and deform to a point where intermolecular forces can no longer hold the structure together, leading to a rapid single-phase-like mixing process. One criticism from this work has been that the experiments were performed on pure components, i.e., normal alkanes from n-heptane to n-hexadecane, and that the complex distillation properties of multi-component fuels may radically affect the conclusions. The lack of knowledge on multicomponent mixtures at the fundamental level limit the usefulness of the aforementioned theoretical calculations to extract interfacial properties under the thermodynamic conditions relevant to diesel spray mixing.

The nature of multi-component fuels is expected to affect the evaporation and mixing processes depending on the thermodynamic conditions inside the chamber at the time of injection. The Coordinating Research Council (CRC) has developed advanced surrogates for diesel combustion research purposes. They produced several multicomponent surrogates with the objective of determining which surrogates best capture the physical and chemical properties of the target diesel fuel and why $[10,11]$. The target fuel, as well as the different surrogate fuels created by the CRC have undergone detailed characterization. These fuels represent the ideal candidates to study the mixing processes of multi-component fuel mixtures under high pressures and temperatures at the microscopic level. In a way similar to our previous campaign on alkane fuels, we applied long-distance microscopy at high speed to resolve in space and track in time the spray-generated droplets under relevant diesel conditions. The ambient conditions were varied to map out the thermodynamic space under which the different mixing regimes were observed, from classical evaporation to diffusive mixing. Time-resolved sequences of the spray processes are presented, and the information extracted from the high-speed visualizations is summarized in pressure and temperature charts highlighting the relationships between mixing behavior and critical properties.

Following the present introduction, the equipment used in this study is described, starting with the high-pressure and high-temperature chamber, followed by the high-speed microscope imaging system, and finishing with the injection system and the different fuels, along with their properties. The results first present an example of the visualization capabilities to resolve and track spray-generated droplets under extreme conditions with alkane fuels. Side-by-side sequences of the classical and diffusive evaporation and mixing processes are shown for the different fuels tested in this study. The results of the observations are then presented on thermodynamic diagrams for the different fuels, showing slight but noticeable variations with respect to mixing behavior.

\section{Experimental apparatus}

The different multi-component fuels and the equipment used to carry out the experimental work are presented in the following paragraphs. The CRC Project AVFL-18a panel $[10,11]$ described the methodology to obtain multicomponent surrogate fuels that match the physico-chemical properties of a reference diesel fuel, as well as the combustion characteristics. The following paragraphs regarding the multi-component fuels briefly describe the methods, but more importantly summarize the information relevant to the present study. As explained in the introduction, previous studies on transcritical mixing from our group used single component fuels, namely normal alkanes. These fuels were used in this study to provide examples as well as baseline to the comparisons with the multi-component fuels detailed thereafter. The CRC formulated a set of four ultralow sulfur diesel surrogate fuels to enable detailed numerical and experimental studies in research engines or dedicated combustion vessels. The different surrogates were designed to match the characteristics of a reference fuel, referred to as CFA target, which corresponds to a sample of the batch A grade no. 2-D S15 diesel emission-certification fuel from Chevron-Philips Chemical Co. The surrogates were formulated with various chemical compounds in order to capture the composition of the target fuel from a hydrocarbon structural standpoint. The objective behind the different surrogate formulations regarding the number and types of components was to identify the appropriate requirements in terms of 
compositional accuracy to effectively match the performance characteristics of the target fuel under different engine operating conditions. The whole process to formulate, blend and characterize the different surrogates was described in great detail in Refs. [10, 11].

Two-dimensional gas chromatography with flame ionization detection performed as part of the target fuel characterization undertaken in Refs. $[10,11]$ revealed that the target fuel contained on the order of 5000 individual components or isomers. The surrogate formulation method relied on a predefined palette of components, as mentioned above. The palette consists of 13 pure compounds, extracted from the normal, cyclo- and branched alkane families, as well as naphtha-aromatic and aromatic species. A regression model was applied to determine the composition that best matches the following parameters: Mole fraction of the different carbon types, derived cetane number, distillation properties and fuel density. The surrogates created through this process, called V0a, V0b, V1 and V2, are made of four, five, eight and nine components, in that order. In this study, we decided to test the CFA target fuel, as well as three of the four surrogates: V0a, V0b and V2. We refer to Ref. [11] for the detailed compositions of the different surrogates. The relevant properties of the target fuel and surrogates are listed in Table 1. Also listed in Table 1 for comparison purposes, are the fluid properties for the normal-alkane fuels used in the present work and in previous studies by the authors [6]: n-heptane, n-dodecane and n-hexadecane.

Table 1. Physico-chemical properties of the various fuels used in this study.

\begin{tabular}{c|ccccccc}
\hline \hline Properties & $\mathrm{n}_{-} \mathrm{C}_{16} \mathrm{H}_{34}$ & $\mathrm{n}-\mathrm{C}_{12} \mathrm{H}_{26}$ & $\mathrm{n}-\mathrm{C}_{7} \mathrm{H}_{16}$ & CFA & V0a & V0b & V2 \\
\hline \hline Number of components & 1 & 1 & 1 & $\sim 5000$ & 4 & 5 & 9 \\
Density $^{1}\left[\mathrm{~kg} / \mathrm{m}^{3}\right]$ & 770 & 750 & 684 & 848 & 818 & 838 & 853 \\
Viscosity $^{2}[\mathrm{mPa} . \mathrm{s}]$ & 2.92 & 1.36 & 0.39 & 2.28 & 2.45 & 2.30 & 2.38 \\
Surface tension $^{3}[\mathrm{mN} / \mathrm{m}]$ & 27.5 & 25.4 & 20.1 & 28.2 & 26.4 & 27.4 & 27.7 \\
Boiling point $^{4,5}[\mathrm{~K}]$ & 554 & 489 & 372 & 345 & 287 & 317 & 344 \\
Critical temperature $^{6}[\mathrm{~K}]$ & 722 & 658 & 540 & - & 440 & 446 & 451 \\
Critical pressure $^{6}[\mathrm{MPa}]$ & 1.43 & 1.82 & 2.73 & - & 1.94 & 2.21 & 2.29
\end{tabular}

${ }^{1}$ Density was measured according to the ASTM D4052 standard method.

2 Kinematic viscosity was measured according to the ASTM D445 standard method.

${ }^{3}$ Surface tension for the multi-component fuels was measured via the plate-method [11].

${ }^{4}$ Boiling point for the surrogate fuels corresponds to the heaviest component property.

${ }^{5}$ Boiling point for the target fuel was measured according to the ASTM D 86 standard method and corresponds to $100 \%$ distillation.

${ }^{6}$ Critical temperature and pressure for the surrogate fuels was estimated from their mole-weighted critical properties.

The fuel sprays were injected in an optically-accessible constant-volume pre-burn vessel with high temperature and pressure capabilities (up to $35 \mathrm{MPa}$ and $1400 \mathrm{~K}$ ). A solenoid-actuated common-rail injector equipped with an axiallydrilled single-hole research nozzle of conical ( $\mathrm{k}$-factor $=1.5), 0.180-\mathrm{mm}$ diameter orifice was used to generate the sprays. The vaporizing and mixing sprays were qualitatively analyzed thanks to a high-speed microscopic system specifically developed for this application. The system consists of a commercial high-speed camera, a long-distance microscope objective and an ultrafast illumination source. The illumination was provided by an LED system designed and developed in our laboratories. A state-of-the-art high-speed CMOS camera was employed to acquire full frame resolution images at 40,000 frames per second (fps). The high-speed camera was equipped with a custom-assembled long-distance microscope objective, producing an effective optical magnification of approximately $8 x$ (digital scale factor $=3.5 \mu \mathrm{m} / \mathrm{pix}$ ). The experimental arrangement, as well as a detailed analysis of the optical performance of the system, paramount in this case, are provided in Ref. [9].

\section{Results and discussion}

As noted earlier, the analysis of the high-speed movies is mostly qualitative. Some efforts have been made to quantify certain parameters that the time-resolved nature of the experiment can extract, such as droplet size or shape as function of time. Size-related information under these conditions is important as it may provide guidance regarding the rate of evaporation, which is key to predict liquid penetration and mixing field in spray and engine simulations. The extracted evaporation rate can also be used to estimate ambient temperature or relative velocity (the difference in velocity between the droplet and the surrounding gas), but such an exercise is complicated in practice because of the many parameters affecting evaporation. Furthermore, accurate species properties are necessary for these results to leverage modelling capabilities, and even the well-characterized multi-component fuels injected in this work are beyond the current numerical capabilities. 
To present the spray system from a global standpoint, we first show a sequence of photographs capturing a relatively large field of view. In this case, the imaging system was set up with an overall magnification of $2 x$, as opposed to the $8 x$ magnification used in the rest of the experiments. Such imaging setup provides a scale factor slightly over $10 \mu \mathrm{m} /$ pixel (compared to the $3.5 \mu \mathrm{m} /$ pix achieved by the system described above), which is still enough to resolve large droplets, especially those generated by the lower injection velocities at the end of injection. The high-speed movies of the image sequences shown in Fig. 1 were acquired at $10 \mathrm{kfps}$. In these sequences, the injector is on the left of the images, and the spray exits the axially-drilled nozzle orifice penetrating horizontally from left to right. A simple intensity normalization has been performed on the image to keep the intensities and contrasts at similar levels across the different sequences. The two time-sequences of Figure 1 show sprays of $n$-hexadecane fuel injected into environments corresponding to different thermodynamic conditions in modern diesel engines. The temperature and pressure for the left panel were $900 \mathrm{~K}$ and $6.0 \mathrm{MPa}$, respectively; the sequence on the right corresponds to an ambient held at $1200 \mathrm{~K}$ temperature and 10.7 MPa pressure. The timing shown at the top left corner is given relative to the end of injection and is synchronized for both sequences. The scale of the images is indicated at the bottom left corner of the top photograph.

The image sequences of Fig. 1 show the injection event at different timings from the middle of the injection to 2.4 $\mathrm{ms}$ after the end of injection, and oriented from left to right for two conditions. The corresponding high-speed movies present a clear perspective over the processes, which contrasts with the somewhat limited effect of the still images presented therein. The left photographs present the spray during the injection event, with tiny droplets on the periphery of the spray on the top sequence. It is much more difficult to identify droplets on the bottom images, even though they appear to be comparable to the top sequence in terms of sharpness and clarity. Both sprays present a dark core, highlighting the optical thickness of high pressure sprays. The following images present the droplet field after the end of injection. It appears that the field of droplets decrease in number substantially with time, across the last two images of the sequence.

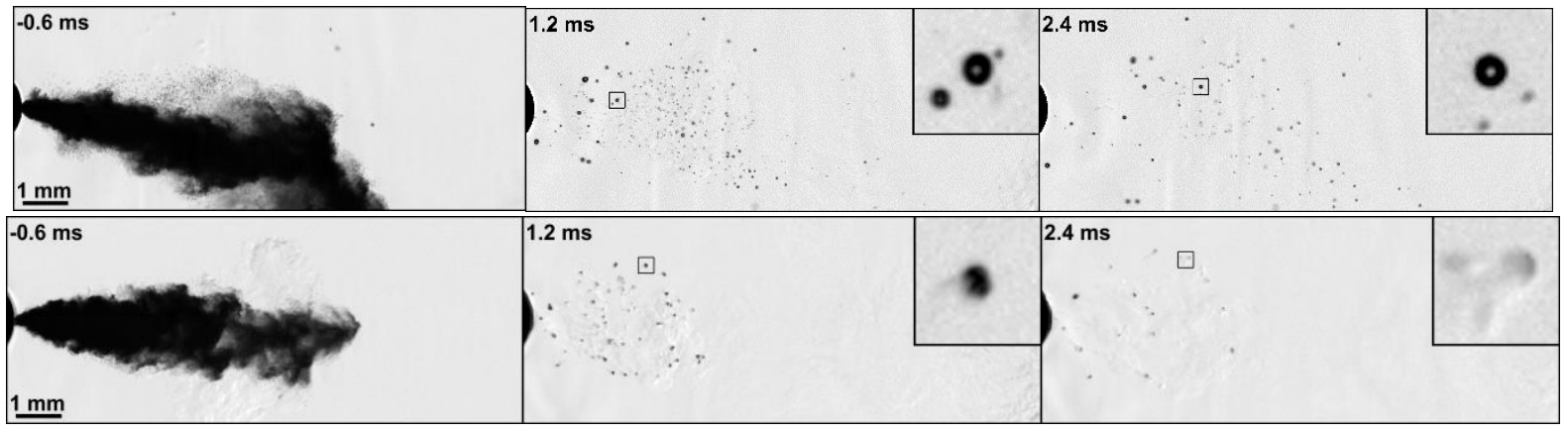

Figure 1: Time-sequence photographs showing the end of injection sequence of $\mathrm{n}$-hexadecane fuel injected under liquid state $(363 \mathrm{~K}, 50 \mathrm{MPa}$ ) into highly heated and pressurized inert environments. Top: Environment at $900 \mathrm{~K}, 6.0 \mathrm{MPa}$ the classical view of liquid breakup and vaporization. Bottom: Environment at $1200 \mathrm{~K}, 10.7 \mathrm{MPa}$ highlighting the effect of high pressure and temperature on mixing.

A magnified droplet, representative of the field, is shown at the top right corner on both sequences to assist with the observations. Both droplets are initially about $50 \mu \mathrm{m}$ in diameter and travel at similar speeds of only a few meters per second. The droplet speed must be compared to the high injection velocities of several hundred meters per second during injection, responsible for droplet size distributions centered around a few micrometers, typical of diesel sprays [12]. The droplet fields produced at the end of injection highlight the differences between the conditions. The droplets of the left panel remain spherical after a brief oscillating period following their formation, and until complete evaporation. The droplets spherical shape reinforces the influence of surface tension, while they decrease in size, until they are no longer successfully identified from the background, corresponding the droplet diameters a little above $10 \mu \mathrm{m}$. Even though it is not readily apparent in the magnified droplet, the evaporation process and associated decrease in droplet diameter is visible through the reduction in droplet number throughout the last two photographs of the sequence. This case exemplifies the classical viewpoint on droplet evaporation, with a mass transfer from liquid to vapor happening on the surface or surrounding of the fluid feature. On the other hand, the droplets produced by the end of injection on the bottom sequence do not remain spherical throughout the images, but deform and mix with the ambient without apparent influence of surface tension. This perspective is clearly shown in the magnified inset, where the photograph taken $1.2 \mathrm{~ms}$ after the end of injection shows a quasispherical droplet that just started vaporizing and deforming, while the following image of the sequence shows the remains of a deformed fluid structure. The optical thickness of the middle photograph contrasts with the relatively clear shadow observed in the last image. The lack of sharpness denotes that the interfacial gradient may not be as 
steep as in the top sequence. These observations are compatible with the broadening of the interface proposed by Dahms and coworkers [2], and described in the introduction. The disruption of the fluid feature and ostensible mass transfer to the surrounding continue in the subsequent images, with only faint disconnected structures remaining in the last image of this sequence. The mass transfer from the fluid structure to the surrounding can be interpreted as a mixing process controlled by aerodynamic forces and molecular diffusion, rather than by the strong intermolecular forces exhibited by surface tension. As such, this process was called diffusive mixing. It can be noted that a number of publications have been referring to this process as supercritical evaporation $[13,14]$, but diffusive mixing appears to be more descriptive of such process.

The two sequences of Fig. 1 also aid as example to detail the criteria employed to qualitatively analyze the various high-speed spray visualizations. Following the descriptions given above for the top sequence, the droplets keep their spherical shape and integrity thanks to surface tension. Most droplets refract light, as shown by the bright spot in their center, denoting that the interface between the droplet and the ambient is sharp, and that the droplet coincides with the imaging system's focal position. Such droplets are tracked to observe whether the optical sharpness of the interface and refraction spot remain, indicating classical evaporation, or degrade, suggesting diffusive mixing. As commented in the previous paragraph, the disappearance of the refraction spot and the observed degradation of the droplet boundaries can be associated with interface broadening and surface tension drop, as suggested by the theoretical calculations carried out by Dahms et al. [2]. Such modifications in the interface state result in lower intermolecular forces, driving the feature to rapidly lose cohesion and disappear in the ambient when subjected to local turbulence and other aerodynamic forces. These observations classify the event as diffusive mixing. Not featured in Fig. 1, transitional mixing was identified when the fluid structures were seen to deform and oscillate, contributing to an accelerated vaporization while the structure remained connected.

Figures 2 to 5 present image sequences for the different fuels under conditions highlighting the two processes observed on Fig. 1 and described above. Similar to Fig. 1, the top sequences of the following figures present the classical evaporating spray viewpoint, showing the droplet formation at the end of injection and the subsequent droplet size reduction until complete evaporation for most of the droplet field. The bottom image panels offer examples of diffusive mixing, where atomization and droplet formation can initially be observed, followed by surface evaporation, until the intermolecular forces can no longer hold the spherical shape of the fluid structures, resulting in deformation and rapid disintegration of the structure to mix with the ambient. The injector is also located on the left side of the images, and the sprays penetrate from left to right. These sprays were injected at pressures between 20 and $25 \mathrm{MPa}$, to keep pressure difference above $10 \mathrm{MPa}$. Unlike Fig. 1, these images were acquired at a higher optical magnification, with a digital resolution on the order of $3.5 \mu \mathrm{m}$ per pixel, allowing better visualization of the atomization process and the generated fluid structures. The main limitation of this optical arrangement is the smaller field of view of approximately $4 \mathrm{~mm}$ in the axial direction (scale displayed at bottom left corner of top image), allowing most droplets to leave the visualized region before being fully vaporized or mixed with the ambient. The acquisition frequency was also increased by a factor of four compared to that of Fig. 1, even though the still images shown thereafter do not highlight the improved temporal resolution. The image panels of Figs. 2 to 5 all present the same timings. All timings reported at the top left corner referred to the end of injection. It should be noted that the ambient conditions shown in Figs. 2 to 5 are provided as examples and should not be used to draw conclusions about the relationship between mixing processes and thermodynamic space for the different fuels. It is important to acknowledge that the image sequences presented therein are limited to describe the processes, and that most of the observations and descriptions surrounding these images were extracted from the original or processed highspeed movies.

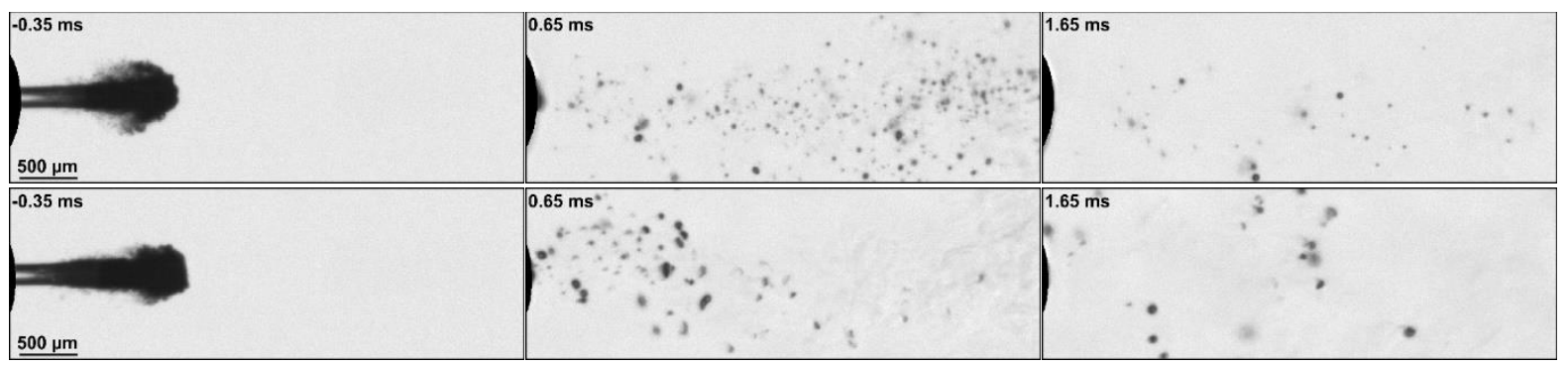

Figure 2: Time-sequence showing the injection, evaporation and mixing processes of sprays of the CFA target fuel into environments at $1000 \mathrm{~K}, 9.2 \mathrm{MPa}$ (left) and 1300, $11.9 \mathrm{MPa}$ (right). The sequence on the left features the classical evaporation, while the right sequence shows signs of diffusive mixing. 


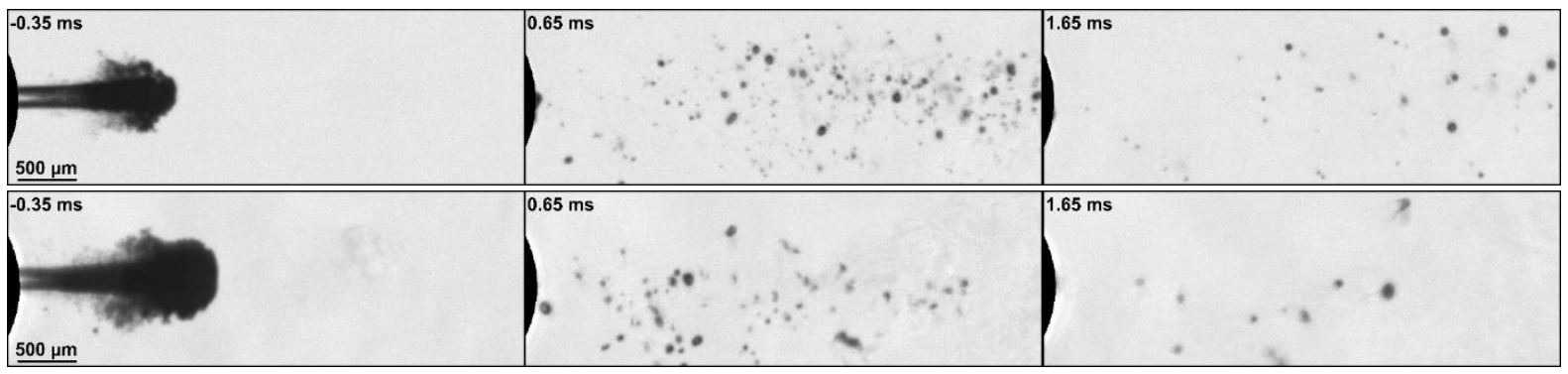

Figure 3: Time-sequence showing the injection, evaporation and mixing processes of V0a surrogate fuel sprays into environments at $900 \mathrm{~K}, 8.2 \mathrm{MPa}$ (left) and 1300, 11.9 MPa (right). The sequence on the left features the classical evaporation, while the right sequence shows signs of diffusive mixing.

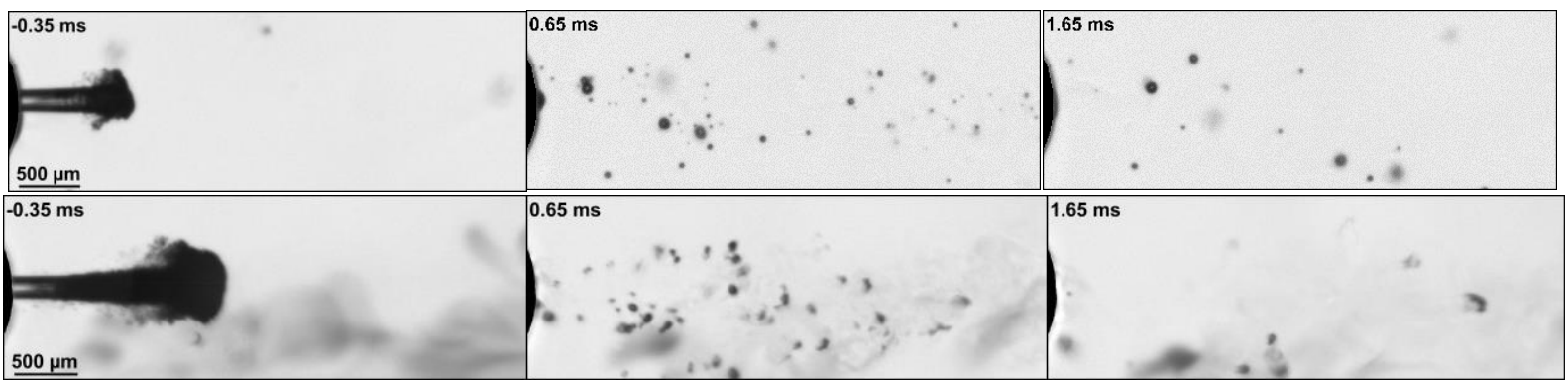

Figure 4: Time-sequence showing the injection, evaporation and mixing processes of V0b surrogate fuel sprays into environments at $900 \mathrm{~K}, 12.2 \mathrm{MPa}$ (left) and 1400, 12.8 MPa (right). The sequence on the left features the classical evaporation, while the right sequence shows signs of diffusive mixing.

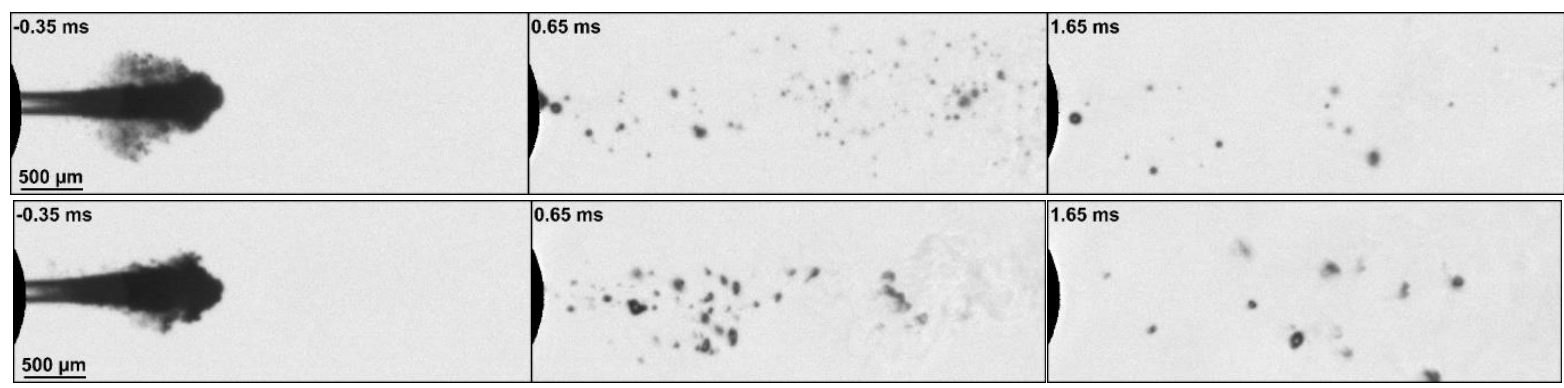

Figure 5: Time-sequence showing the injection, evaporation and mixing processes of $\mathrm{V} 2$ surrogate fuel sprays into environments at $900 \mathrm{~K}, 12.2 \mathrm{MPa}$ (left) and 1400, $12.8 \mathrm{MPa}$ (right). The sequence on the left features the classical evaporation, while the right sequence shows signs of diffusive mixing.

As mentioned earlier for Fig. 1, the sequences presented in Figs. 2 to 5 are synchronized together with respect to the end of injection. The first image of the sequence shows the developing spray, acquired shortly after the start of injection, corresponding to approximately $350 \mu$ s before the end of injection in these examples. Paying attention to the periphery of the plume, small fluid structures can be observed on both first images of most sequences. It is however difficult to assess whether these features are spherical or deformed due to their small dimensions, and possible motion blurring. Fluid features are in general visible on the spray periphery throughout the injection event, but the short life-times and small sizes render the analysis complicated, as detailed earlier. The spray rapidly dissipates in the next images of the various sequences, with the faster moving features exiting the visualized domain, while the slower structures are left behind. The remaining images in the sequences highlight the differences between the mixing processes previously described alongside Fig. 1, with spherical droplets decreasing in size in the top sequences, and deforming fluid features eventually mixing with the ambient via diffusion on the bottom images. It should be noted that the ambient conditions of the lower panels are somewhat above the transition limit between classical/transitional mixing and diffusive mixing. These elevated temperature and pressure conditions are necessary to ensure that the fluid structures are deforming and mixing with the ambient with substantially reduced intermolecular forces within the 4-mm long field of view.

In order to summarize the experiments and compare the different fuels in the scheme of the diffusive mixing process, the tests have been classified on pressure - temperature diagrams. Figure 6 shows these diagrams for the four 
multi-component fuels tested in this study. All diagrams are set to the same limits to facilitate comparisons across the different fuels. White symbols represent conditions where only classical evaporation and mixing was observed, while the black symbols correspond to conditions where diffusive mixing was identified. The transitional mixing regime is identified by grey markers.
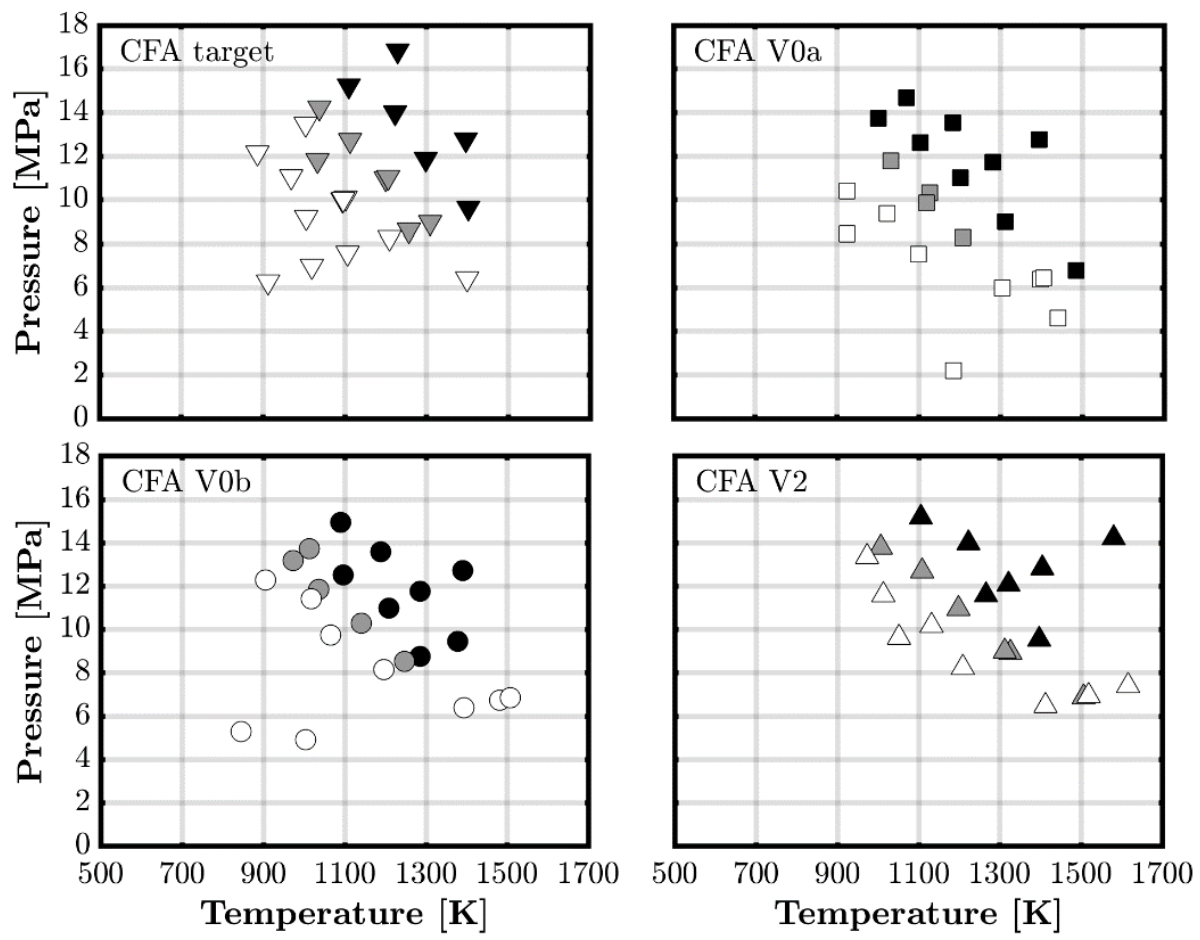

Figure 6: Pressure-temperature diagrams showing the test points for each of the four multi-component fuels. The white symbols represent test conditions where only classical evaporation is observed; the gray markers represent conditions showing transitional mixing; the black symbols represent test conditions where the fluid exhibits diffusive mixing.

The relatively large thermodynamic space of the diagrams in Fig. 6 does not highlight the differences between the fuels. At the same time, it is not surprising that the fuels show similarities because the surrogates were produced to match certain characteristics of the CFA target fuel. It should be noted though, that matching the critical properties was not part of the objectives of the formulation process. Despite these apparent similarities, a closer look at the black symbols shows that CFA target fuel is best matched by V2, while V0a and V0b present relatively similar behavior on the pressure - temperature space. As noted after Table 2, the closest surrogate to CFA target in terms of physico-chemical properties in this study is the nine-component V2 surrogate fuel. Characteristics such as density and boiling point are matched within $1 \%$ between these two fuels. These two fuels present transcritical mixing behavior at higher temperature and pressure regions in the thermodynamic charts of Fig. 6 compared to the other two fuels. It should be noted that the ambient pressures and temperatures where diffusive mixing has been observed are typical conditions near or past top-dead-center for modern diesel engines. These tests therefore confirm our previous findings about mixing properties under realistic thermodynamic conditions on singlecomponent fuels.

\section{Conclusions}

Microscopic observations of diesel sprays injected in high pressure and temperature environments have been carried out to understand the vaporization and mixing processes of multi-component fuels above the critical point. Three multi-component diesel surrogate fuels were used, in addition to a well-characterized commercial diesel target fuel. The surrogate fuels were compared to the target fuel by means of detailed high-speed visualizations of spray processes in the near-nozzle region.

As previously observed for the alkane sprays, the four multi-component fuel sprays present three mixing processes: classical evaporation, transitional mixing and diffusive mixing. These experiments demonstrate that diesel fuels are also subject to transcritical mixing, where the effects of surface tension are significantly reduced to allow the fluid structures to rapidly mix with the surrounding. Differences between the multi-component fuels have been observed when plotting the regime classification on pressure - temperature diagrams. The thermodynamic spaces where the different mixing regimes were identified shift from one fuel to another, and agree with the particular fuel composition 
and physico-chemical properties. Interestingly, the most complex surrogate formulation proved to be the best match for the target fuel in these experiments, despite the fact that critical properties were not accounted for during the formulation. Future efforts will focus on determining the fluid structure lifetime under the different regimes, to establish a relationship between thermodynamic properties and vaporization/mixing rate for single and multicomponent fuels.

\section{Acknowledgements}

This work was supported by the UK's Engineering and Physical Science Research Council [grant number EP/K020528/1]. The authors gratefully acknowledge Coordinating Research Council Project AVFL-18a for formulating, characterizing, and providing the target and surrogate fuels used in this study. This study was performed at the Combustion Research Facility, Sandia National Laboratories is a multi-mission laboratory managed and operated by National Technology and Engineering Solutions of Sandia, LLC., a wholly owned subsidiary of Honeywell International, Inc., for the U.S. Department of Energy's National Nuclear Security Administration under contract DE-NA0003525.

\section{References}

[1] ExxonMobil. The outlook for energy: A view to 2040. Outlook Series, 2015.

[2] R. N. Dahms, J. Manin, L. M. Pickett, and J. C. Oefelein. Understanding high-pressure gas-liquid interface phenomena in diesel engines. Proceedings of the Combustion Institute, 34(1):1667-1675, 2013.

[3] R. N. Dahms. Gradient theory simulations of pure fluid interfaces using a generalized expression for influence parameters and a Helmholtz energy equation of state for fundamentally consistent two-phase calculations. Journal of colloid and interface science, 445:48-59, 2015.

[4] N. Zong and V. Yang. Cryogenic fluid jets and mixing layers in transcritical and supercritical environments. Combustion science and technology, 178(1-3):193-227, 2006.

[5] B. Chehroudi. Recent experimental efforts on high-pressure supercritical injection for liquid rockets and their implications. International Journal of Aerospace Engineering, 2012, 2012.

[6] C. Crua, J. Manin, and L. M. Pickett. On the transcritical mixing of fuels at diesel engine conditions. Submitted to Fuel, 2017.

[7] Z. Falgout, M. Rahm, Z. Wang, and M. Linne. Evidence for supercritical mixing layers in the ECN Spray A. Proc. Combust. Inst., 35(2):1579-1586, 2015.

[8] M. Wensing, T. Vogel, and G. Gotz. Transition of diesel spray to a supercritical state under engine conditions. International Journal of Engine Research, 17(1):108-119, 2016.

[9] J. Manin, M. Bardi, L. M. Pickett, R. N. Dahms, and J. C. Oefelein. Microscopic investigation of the atomization and mixing processes of diesel sprays injected into high pressure and temperature environments. Fuel, 134:531-543, 2014.

[10] C. J. Mueller, W. J. Cannella, T. J. Bruno, B. Bunting, H. D. Dettman, J. A. Franz, M. L. Huber, M. Natarajan, W. J. Pitz, M. A. Ratcliff, and Others. Methodology for formulating diesel surrogate fuels with accurate compositional, ignition-quality, and volatility characteristics. Energy \& Fuels, 26(6):3284-3303, 2012.

[11] C. J. Mueller, W. J. Cannella, J. T. Bays, T. J. Bruno, K. DeFabio, H. D. Dettman, R. M. Gieleciak, M. L. Huber, C.-B. Kweon, S. S. McConnell, W. J. Pitz, and M. A. Ratcliff. Diesel surrogate fuels for engine testing and chemical-kinetic modeling: Compositions and properties. Energy \& Fuels, 30(2):1445-1461, 2016.

[12] C. Crua, G. de Sercey, M. R. Heikal, and Gold M. Dropsizing of near-nozzle Diesel and RME sprays by microscopic imaging. ICLASS 2012, Heidelberg, Germany, 2012.

[13] S. D. Givler and J. Abraham. Supercritical droplet vaporization and combustion studies. Prog. Energ. Combust., 22(1):1-28, 1996.

[14] G. S. Zhu and S. K. Aggarwal. Transient supercritical droplet evaporation with emphasis on the effects of equation of state. International Journal of Heat and Mass Transfer, 43(7):1157-1171, 2000. 